\title{
Predictive and Prognostic Factors in Ovarian and Uterine Carcinosarcomas
}

\author{
İrfan Cicin ${ }^{1}$, Tahsin Özatl1², Esma Türkmen ${ }^{1}$, Türkan Özturk ${ }^{3}$, Melike Özçelik ${ }^{4}$, Devrim Çabuk $^{5}$, \\ Ayşe Gökdurnalıㄹ, Özlem Balvan ${ }^{4}$, Yaşar Yıldız ${ }^{6}$, Metin Şeker ${ }^{7}$, Nuriye Özdemir ${ }^{8}$, Burcu Yapar ${ }^{9}$, \\ Özgür Tanrıverdi ${ }^{10}$, Yusuf Günaydin ${ }^{11}$, Serkan Menekşse ${ }^{12}$, Berna Öksüzoğlu ${ }^{2}$, Asude Aksoy ${ }^{13}$, \\ Bülent Erdogan ${ }^{1}$, M. Bekir Hacıoglu ${ }^{1}$, Erkan Arpaci ${ }^{14}$, Alper Sevinç ${ }^{15}$
}

${ }^{1}$ Department of Medical Oncology, Trakya University School of Medicine, Edirne, Turkey
${ }^{2}$ Department of Medical Oncology, Ankara Dr. Abdurrahman Yurtaslan Oncology Training and Research Hospital, Ankara, Turkey
${ }^{3}$ Department of Medical Oncology, Karadeniz Technical University School of Medicine, Trabzon, Turkey
${ }^{4}$ Department of Medical Oncology, Dr. Lütfi Kurdar Research and Training Hospital, Istanbul, Turkey.
${ }^{5}$ Department of Medical Oncology, Kocaeli University School of Medicine, Kocaeli, Turkey
${ }^{6}$ Department of Medical Oncology, Atatürk Research and Training Hospital, İzmir, Turkey
${ }^{7}$ Department of Medical Oncology, Cumhuriyet University School of Medicine, Sivas, Turkey
${ }^{8}$ Department of Medical Oncology, Ankara Numune Research and Training Hospital, Ankara, Turkey
${ }^{9}$ Department of Medical Oncology, Pamukkale University School of Medicine, Denizli, Turkey
${ }^{10}$ Department of Medical Oncology, Muğla University School of Medicine, Muğla, Turkey
${ }^{11}$ Department of Medical Oncology, Gazi University School of Medicine, Ankara, Turkey
${ }^{12}$ Department of Medical Oncology, Celal Bayar University School of Medicine, Manisa, Turkey
${ }^{13}$ Department of Medical Oncology, İnönü University School of Medicine, Malatya, Turkey
${ }^{14}$ Department of Medical Oncology, Sakarya Research and Training Hospital, Sakarya, Turkey
${ }^{15}$ Department of Medical Oncology, Gaziantep University School of Medicine, Gaziantep, Turkey

Background: Prognostic factors and the standard treatment approach for gynaecological carcinosarcomas have not yet been clearly defined. Although carcinosarcomas are more aggressive than pure epithelial tumours, they are treated similarly. Serous/clear cell and endometrioid components may be predictive factors for the efficacy of adjuvant chemotherapy (CT) or radiotherapy (RT) or RT in patients with uterine and ovarian carcinosarcomas. Heterologous carcinosarcomas may benefit more from adjuvant CT.

Aims: We aimed to define the prognostic and predictive factors associated with treatment options in ovarian (OCS) and uterine carcinosarcoma (UCS).

Study Design: Retrospective cross-sectional study Methods: We retrospectively reviewed the medical records of patients with ovarian and uterine carcinosarcoma from 2000 to 2013, and 127 women were includ- ed in this study (24 ovarian and 103 uterine). Patients admitted to seventeen oncology centres in Turkey between 2000 and December 2013 with a histologically proven diagnosis of uterine carcinosarcoma with FIGO 2009 stage I-III and patients with sufficient data obtained from well-kept medical records were included in this study. Stage IV tumours were excluded. The patient records were retrospectively reviewed. Data from 104 patients were evaluated for this study.

Results: Age ( $\geq 70$ years) was a poor prognostic factor for UCS ( $p=0.036)$. Pelvic \pm para aortic lymph node dissection did not affect overall survival (OS) $(p=0.35$ ). Macroscopic residual disease was related with OS $(p<0.01)$. The median OS was significantly longer in stage I-II patients than stage III patients $(\mathrm{p}=0.03)$. Adjuvant treatment improved OS $(p=0.013)$. Adjuvant radiotherapy tended to increase the median OS

This study has been presented at the $11^{\text {th }}$ National Medical Oncology Congress, 17-21 September 2014, Turkish Republic of Northern Cyprus.

Address for Correspondence: Dr. Bülent Erdoğan, Department of Medical Oncology, Trakya University School of Medicine, Edirne, Turkey

Phone: +905052137335 e-mail: berdoga@hotmail.com

Received: 29 September $2015 \quad$ Accepted: 18 April $2016 \bullet$ DOI: $10.5152 /$ balkanmedj.2016.151268

Available at www.balkanmedicaljournal.org 
$(p=0.075)$. However, this tendency was observed in UCS $(p=0.08)$ rather than OCS $(p=0.6)$. Adjuvant chemotherapy had no effect on OS $(p=0.15)$.Adjuvant radiotherapy significantly prolonged the median OS in patients with endometrioid component $(p=0.034)$. A serous/clear cell component was a negative prognostic factor $(\mathrm{p}=0.035)$. Patients with serous/clear cell histology for whom adjuvant chemotherapy was applied had significantly longer OS $(p=0.019)$, and there was no beneficial effect of adjuvant radiotherapy $(\mathrm{p}=0.4)$. Adjuvant chemotherapy was effective in heterologous tumours $(\mathrm{p}=0.026)$. In multivariate analysis, the stage and chemotherapy were prognostic factors for all patients. Age was an independent prognostic factor for UCS. However, serous/clear cell histology and radiotherapy tended to be significant prognostic factors.

Conclusion: The primary location, the histological type of sarcomatous and the epithelial component may be predictive factors for the efficacy of chemotherapy or radiotherapy in UCS and OCS.

Keywords: Carcinosarcoma, ovary, uterine, predictive factors, prognostic factors
Carcinosarcomas are rare gynecological tumors; approximately $2-5 \%$ of all uterine and ovarian cancers are carcinosarcomas. Primary peritoneal, vaginal and vulvar carcinosarcomas are extremely rare tumors. Carcinosarcomas are composed of carcinomatous and sarcomatous components. The subtypes are defined as homologous (the presence of sarcomatous component originating from the mesenchymal content of the primary tumor location) and heterologous (the presence of sarcomatous component not originating from the mesenchymal content of the primary tumor location) according to the sarcomatous component (1). Histogenetic analyses indicated that these tumors originate from epithelial tissue; however, the characteristics and course of the disease are different from epithelial tumors $(2,3)$. Gynecological carcinosarcomas are highly aggressive neoplasms with poor prognosis $(4,5)$. In previous studies, various prognostic factors have been defined; however, there is no consensus about these factors. Treatment approaches have not been clearly identified for epithelial uterine cancers. The predictive factors for response to treatment are also not known. However, data about uterine carcinosarcoma, due to the rarity of these tumors, are based on retrospective experiences rather than prospective studies. The number of patients was low in the published studies. In this multicenter retrospective analysis, we aimed to define the clinical features and prognostic and predictive factors for treatment options with a larger sample size. The most important difference from the published studies are that it was a multicenter study, had a larger sample size, more treatment modalities and comparable primary origins.

\section{MATERIALS AND METHODS}

The present study was designed as a retrospective analysis of a primary uterine and ovarian carcinosarcoma patient cohort. $\mathrm{Pa}-$ tients admitted to seventeen oncology centers between 2000 and December 2013 with a histologically proven diagnosis of uter- ine carcinosarcoma with FIGO 2009 stage I-III and patients with sufficient data obtained from well-kept medical records were included in this study. Stage IV tumors were excluded. The patient records were retrospectively reviewed after approval of the study by the University Ethical Committee. Data from 104 patients were evaluated for this study. Data were collected from the centers with an online standardized form.

Patient data including age at diagnosis, parity, menopausal status, the date of diagnosis, the date and location of disease recurrence, the death date or the last contact date, the applied surgical procedure, lymph node dissection, and the epithelial and sarcomatous components of the tumor were recorded from patient charts.

Macroscopic residual disease was defined as the presence of $\geq 2 \mathrm{~cm}$ of tumor remnant after surgery. Post-surgical treatment was recorded as observation (OBS), radiotherapy (RT) alone (brachytherapy, whole pelvic, or combination), chemotherapy (CT) alone and radio-chemotherapy (RCT) (concomitant, sequential).

\section{Statistical methods}

Normally distributed numerical data were tested with the one-sample Kolmogorov-Smirnov test. Numeric variables with normal distribution were compared with Student's t-test, and those that were not normally distributed were compared with the Mann-Whitney U test. Categorical variables were compared using the chi-squared test. Overall survival (OS) was accepted as the period from diagnosis to death. The survival estimates were calculated using the Kaplan-Meier method, and survival estimates between groups were compared with the log-rank test. Statistical analysis was performed using SPSS 20.0 (SPSS; IBM Corp.; New York, USA). A p value of $<0.05$ was considered to indicate statistical significance. Factors that had a $p$ value of $\leq 0.15$ in the univariate analysis were examined in the multivariate analysis (Cox regression). 
TABLE 1. The demographical, clinical and pathological features of ovarian and uterine carcinosarcomas

\begin{tabular}{lccc}
\hline & Ovary & Uterine & $\mathrm{p}$ \\
\hline Age & $58 \pm 12$ & $62 \pm 11$ & 0.10 \\
Parity & 3 & 4 & 0.19 \\
Menopause status & & & \\
$\quad$ Premenopausal & 6 & 12 & 0.11 \\
Postmenopausal & 18 & 91 &
\end{tabular}

\section{Subtype}

Heterologous

Homologous

Unknown

Epithelial subtypes

Mucinous

Serous

Clear cell

Endometrioid

Squamous

Undifferentiated

Unknown

Sarcomatous subtypes

Stromal sarcoma
Leiomyosarcoma
Undifferentiated
Chondrosarcoma
Rhabdomyosarcoma
Liposarcoma
Fibrosarcoma
MFH
Biphenotypic
Unknown

Mean tumor diameter

Stage

I
II
III

ECOG status

\begin{tabular}{cccc}
0 & 10 & 33 & \\
1 & 20 & 59 & \\
2 & 2 & 10 & 0.12 \\
3 & 2 & 1 & \\
4 & - & - & \\
\hline
\end{tabular}

MFH: malign fibrous histiositosarcoma

\section{RESULTS}

One hundred and twenty seven women were eligible for the study. The median age was 61 (range 26-85) years. One hundred and nine (86\%) of the patients were postmenopausal, and $103(81.1 \%)$ women had uterine carcinosarcoma (UCS) (94 corpus uteri + eight cervix uteri + one vaginal carcinosarcoma). The sarcomatous component was homologous in 50 (39.4\%) patients and heterologous in $54(52.5 \%)$ patients. The sarcomatous component was not reported in the pathology reports of twenty-three (18.1\%) patients. Leiomyosarcoma was the most common sarcomatous component in the UCS; the second most common sarcomatous component was rhabdomyosarcoma. The most common epithelial component in the UCS were serous papillary carcinoma and endometrioid carcinoma, respectively. The demographic and clinical features of the patients are presented in Table 1.

TABLE 2. Prognostic factors for ovarian and uterine carcinosarcomas

\begin{tabular}{|c|c|c|c|c|c|}
\hline & \multicolumn{3}{|c|}{ Univariate analysis } & \multicolumn{2}{|c|}{ Multivariate analysis } \\
\hline & Ovarian & Uterine & Overall & Uterine & Overall \\
\hline \multicolumn{6}{|l|}{ Age } \\
\hline$<70$ & 0.12 & 0.036 & 0.23 & 0.02 & - \\
\hline \multicolumn{6}{|l|}{$\geq 70$} \\
\hline \multicolumn{6}{|c|}{ Menopause status } \\
\hline $\begin{array}{l}\text { Premenopausal } \\
\text { Postmenopausal }\end{array}$ & 0.2 & 0.28 & 0.26 & - & - \\
\hline \multicolumn{6}{|l|}{ Surgery } \\
\hline LND & 0.31 & 0.65 & 0.75 & - & - \\
\hline \multicolumn{6}{|l|}{ No LND } \\
\hline \multicolumn{6}{|l|}{ Stage } \\
\hline I-II & 0.008 & 0.27 & 0.03 & - & 0.05 \\
\hline \multicolumn{6}{|l|}{ III } \\
\hline \multicolumn{6}{|c|}{ Epithelial subtype } \\
\hline Endometrioid & 0.33 & 0.36 & 0.26 & - & - \\
\hline \multicolumn{6}{|l|}{ Nonendometrioid } \\
\hline \multicolumn{6}{|l|}{ Epithelialsubtype } \\
\hline Serous/clear & 0.5 & 0.035 & 0.033 & 0.071 & 0.088 \\
\hline \multicolumn{6}{|l|}{ Non-serous/clear } \\
\hline \multicolumn{6}{|c|}{ Sarcomatous subtype } \\
\hline Heterolog & 0.35 & 0.5 & 0.62 & - & - \\
\hline \multicolumn{6}{|l|}{ Homolog } \\
\hline Residual tumor & 0.021 & 0.12 & 0.01 & 0.72 & 0.24 \\
\hline Chemotherapy & 0.9 & 0.12 & 0.15 & 1.4 & 0.041 \\
\hline Radiotherapy & 0.6 & 0.08 & 0.075 & 0.058 & 0.36 \\
\hline
\end{tabular}

LND: lymph node dissection 
The median OS was 26.3 (95\% CI, 17.9-34.7) months. Menopausal status was not a prognostic factor. Advanced age ( $<70$ years vs. $\geq 70$ years) was found to be a prognostic factor (Table 2).

One hundred and twenty-three patients underwent surgery. The surgical procedures are summarized in Table 3. The OS was similar in patients who underwent pelvic \pm Para aortic lymph node dissection and in patients who did not undergo lymph node dissection $(\mathrm{p}=0.75$ ). Macroscopic residual disease after surgery was significantly related with OS $[32.5(95 \%$ CI, 11.1-27.8) months vs. 19.5 (95\% CI, 23.9-41.2) months, $\mathrm{p}=0.003]$.

Patients with stage I and stage II were evaluated together because five of the seventeen patients in stage II had died. The OS was longer for patients with stage I-II compared to patients with stage III $(\mathrm{p}=0.03)$. Stage III OCS patients had poorer survival than stage III UCS patients, although Stage I-II OCS patients had better OS results than the stage I-II UCS patients $(\mathrm{p}=0.005$, Figure 1$)$.
Patients who underwent adjuvant treatment had longer median OS than patients who did not [32.5 (95\% CI, 23.341.8) months vs. 18.3 (95\% CI, 3.9-32.7 months), $\mathrm{p}=0.013$ ] (Figure 2a).

Adjuvant RT was applied in 54 of 127 patients. Adjuvant RT had a tendency to increase the median OS [32.5 (95\% CI, 23.641.5) months vs. 20.7 (95\% CI, 12.2-29.1) months, $\mathrm{P}=0.075]$. However, this tendency was more prominent in UCS patients $(\mathrm{p}=0.08)$ compared to OCS patients $(\mathrm{P}=0.6)$.

Adjuvant chemotherapy ( $89.2 \%$ platinum based) was given to 83 of 127 patients. Adjuvant chemotherapy had no impact on the median OS $(\mathrm{p}=0.15)$ in univariate analysis. Patients with UCS and OCS who received adjuvant CT had similar OS $(\mathrm{p}=0.64)$. Adjuvant chemotherapy did not improve the median OS in UCS patients $(\mathrm{p}=0.12)$. The overall survival was only 3.8 months in three patients with OCS who did not receive adjuvant chemotherapy. However, two of these patients did not receive chemotherapy due to post-operative complications and one was lost to follow up.

TABLE 3. Predictive factors for radiotherapy and chemotherapy in ovarian and uterine carcinosarcomas

\begin{tabular}{|c|c|c|c|c|c|c|}
\hline & \multicolumn{3}{|c|}{ RT } & \multicolumn{3}{|c|}{$\mathrm{CT}$} \\
\hline & Yes & No & $\mathrm{p}$ & Yes & No & $\mathrm{p}$ \\
\hline \multicolumn{7}{|l|}{ Age } \\
\hline$\geq 70$ & $27.1(16.8-37.5)$ & $13.9(4.3-23.5)$ & 0.4 & $27.1(10.6-43.7)$ & $26.3(4.4-48.2)$ & 0.3 \\
\hline \multicolumn{7}{|l|}{ Stage } \\
\hline III & $22.3(15.5-29.0)$ & $18.3(10.3-26.3)$ & 0.2 & $22.8(12.9-31.7)$ & $12.0(2.5-21.5)$ & 0.001 \\
\hline \multicolumn{7}{|l|}{ Primary site } \\
\hline Ovary & $30.2(6.5-54.0)$ & $19.4(8.0-31.0)$ & 0.6 & $20.7(2.2-39.1)$ & 3.8 (not reached) & 0.9 \\
\hline Uterine & $33.1(22.0-44.1)$ & $24.0(15.7-32.2)$ & 0.08 & $27.1(14.2-40.1)$ & $26.3(20.0-32.7)$ & 0.12 \\
\hline \multicolumn{7}{|l|}{ Epithelial subtype } \\
\hline Endometrioid & $67.4(29.8-105.1)$ & $34.6(10.3-58.9)$ & 0.034 & $34.7(19.1-50.3)$ & $45.6(6.1-85.2)$ & 0.9 \\
\hline Non-endometrioid & $27.1(16.1-38.2)$ & $20.2(11.4-29.0)$ & 0.4 & $25.8(0-53.2)$ & $22.3(14.0-29.6)$ & 0.064 \\
\hline \multicolumn{7}{|l|}{ Epithelial subtype } \\
\hline Serous/clear & $16.0(14.5-17.6)$ & $20.2(17.0-23.5)$ & 0.4 & $20.7(12.6-28.7)$ & $9.6(0.4-18.8)$ & 0.019 \\
\hline Non-serous/clear & $40.5(0-84.4)$ & $25.8(11.7-39.6)$ & 0.053 & $33.5(16.1-49.0)$ & $32.3(16.9-49.2)$ & 0.3 \\
\hline \multicolumn{7}{|l|}{ Surgery } \\
\hline LND & $30.2(21.4-39.1)$ & $18.1(10.6-25.6)$ & 0.082 & $23.1(12.8-33.5)$ & $26.3(8.8-43.9)$ & 0.2 \\
\hline
\end{tabular}

RT: radiotherapy; CT: chemotherapy; LND: lymph node dissection 
Patients who received adjuvant RT or RCT had a longer median OS than patients who did not receive adjuvant treatment $(p=0.027$ and $p=0.021$, respectively). The median OS of the patients who received adjuvant $\mathrm{CT}$ had a tendency to be longer than the patient in the OBS arm $(p=0.076)$. The overall survivals for patients in the adjuvant RT, RCT and CT arms were similar (Figure 2b).

Numerically, the epithelial component was associated with the longest median OS was endometrioid histology (data not presented). When the patients were stratified as endometrioid and non-endometrioid by epithelial component, the endometrioid component was not a prognostic factor for both the OCS $(p=0.33)$ and UCS $(p=0.36)$ groups. Adjuvant RT significantly prolonged the median OS in patients with endometrioid component (0.034) (Figure 3a). Adjuvant CT had no effect on the survival of patients with endometrioid component. In patients with non-endometrioid component, while adjuvant RT had no beneficial effect on survival $(p=0.4)$ (Figure $3 b)$, adjuvant CT had a tendency to be beneficial $(\mathrm{p}=0.064)$.

Numerically, the epithelial component associated with the shortest median OS was serous/clear cell histology (data not presented). When the patients were stratified as serous/clear cell and non-serous/clear cell component, serous/clear cell histology was found to be a negative prognostic factor $(\mathrm{p}=0.035)$. This difference was observed in UCS patients $(\mathrm{p}=0.033)$ but not in OCS patients $(\mathrm{p}=0.5)$. Patients with serous/clear cell histology who received adjuvant CT had significantly longer median OS $(\mathrm{P}=0.019)$ (Figure $3 \mathrm{c})$, and there was no beneficial effect of adjuvant RT ( $\mathrm{p}=0.4)$ (Table 3 ). In patients with nonserous/clear cell component, adjuvant CT had no beneficial effect on survival $(p=0.4)$ (Figure $3 d$ ).

There was no relationship between the sarcomatous sub-

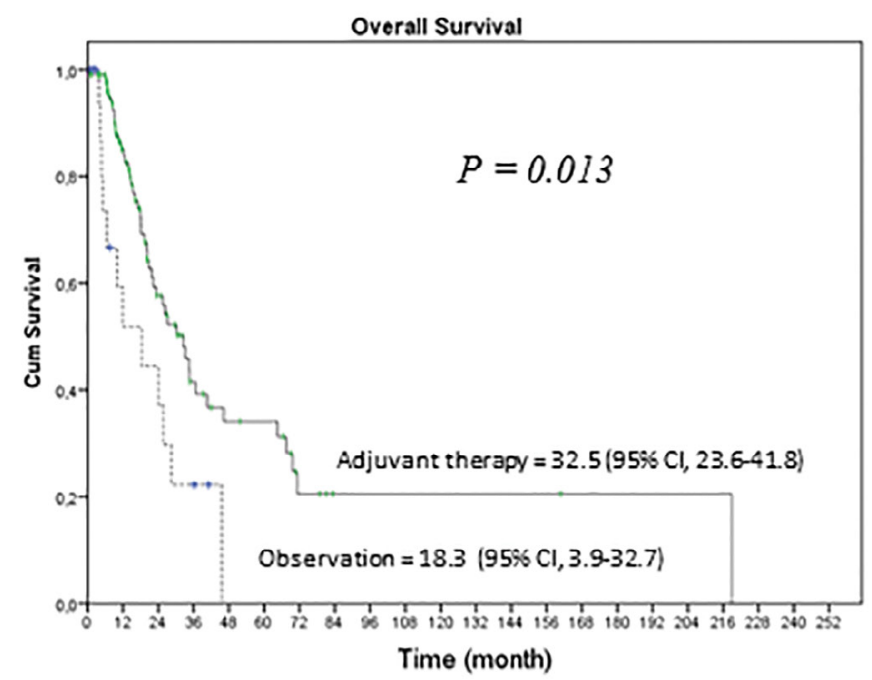

types and the median OS ( $p=0.62)$. The efficacy of RT was not similar among the sarcomatous subtypes. However, adjuvant CT was beneficial in patients with heterologous subtypes $(\mathrm{p}=0.026)$ (Table 3$)$.

In multivariate analysis, the disease stage and CT were independent prognostic factors for all patients, and serous/clear cell histology tended to be a significant factor. Age was an independent prognostic factor for UCS patients. Serous/clear cell histology and RT had trends towards being significant prognostic factors (Table 2). Patients with OCS could not be evaluated with multivariate analysis due to the low patient and event numbers.

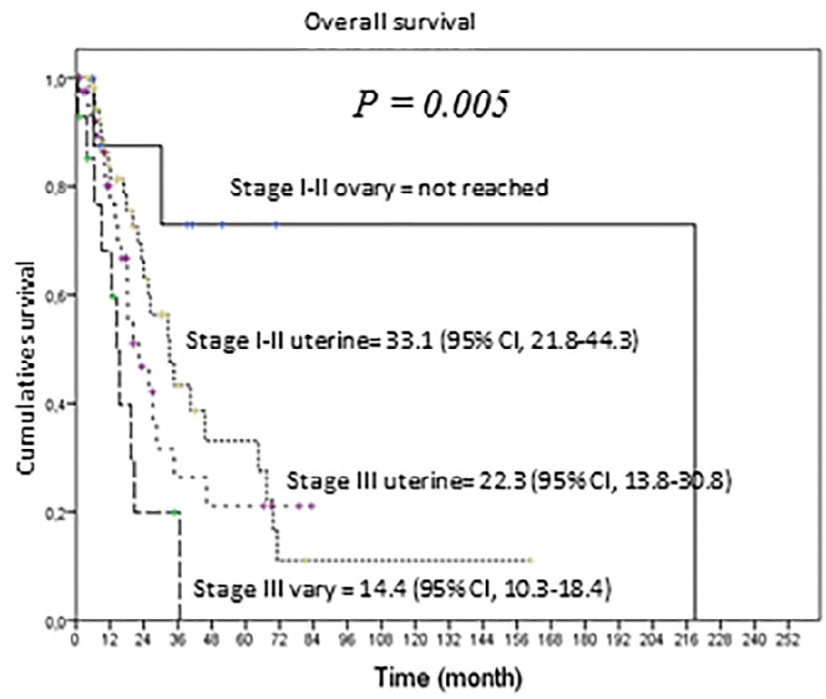

FIG. 1. Overall survivals of stage I-II and stage III patients with uterine and ovarian carcinosarcomas.

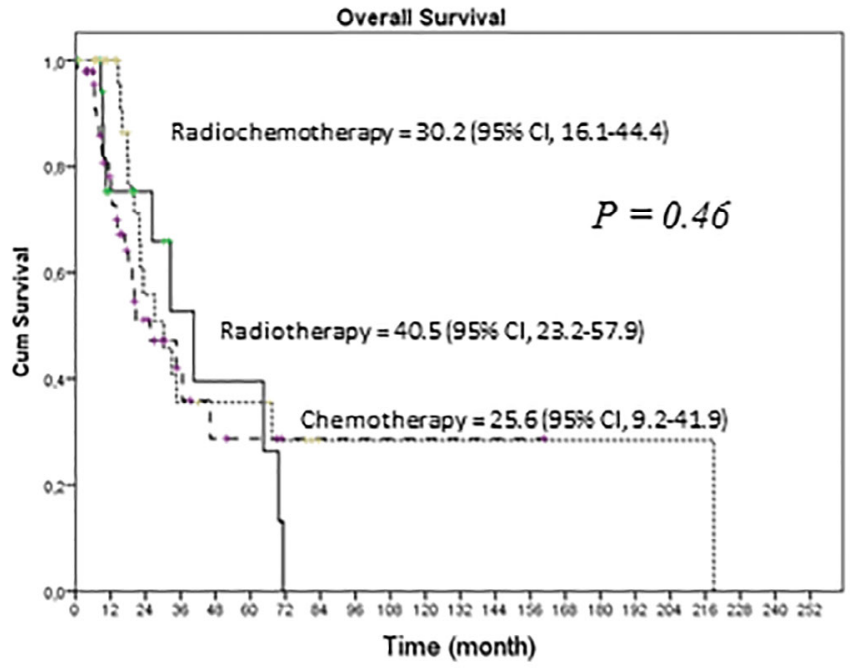

FIG. 2. a-b. Overall survivals of the patients who received adjuvant therapy and those who did not receive adjuvant therapy (a). The effect of adjuvant therapies on uterine and ovarian carcinosarcomas (b). 


\section{DISCUSSION}

Prognostic factors and the standard treatment approach for gynecological carcinosarcomas have not yet been clearly defined. Although carcinosarcomas are more aggressive than pure epithelial tumors, they are treated similarly. It is known that they are more sensitive to ifosfamide than epithelial tumors and insensitive to anthracyclines compared to sarcomas $(4,5)$. Erickson et al. (6) suggested that black race independently predicts worse survival in UCS. However, the predictive factors related to treatment success are not clearly defined. In this current study, we aimed to define the clinical and pathologic features, and the prognostic and predictive factors related with treatment.

a

Overall Survival

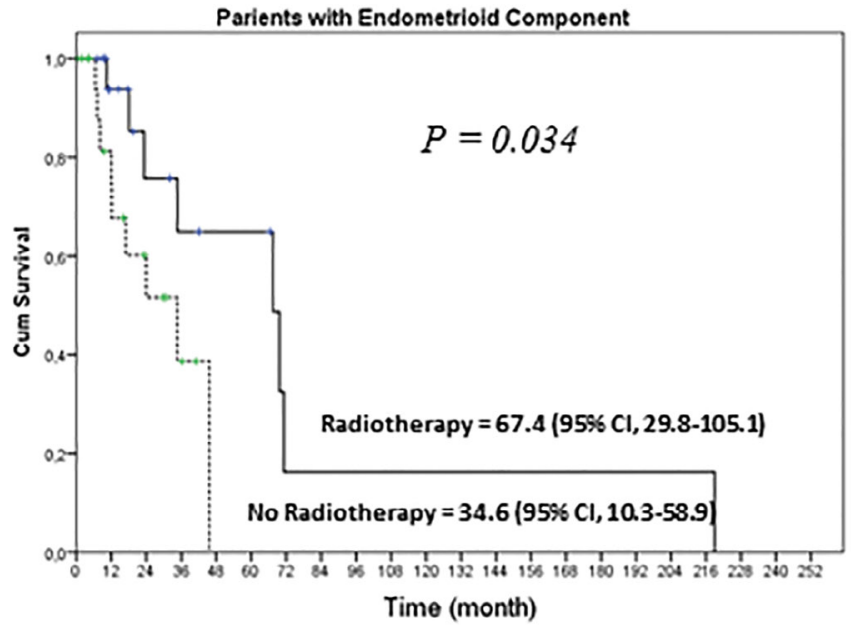

C

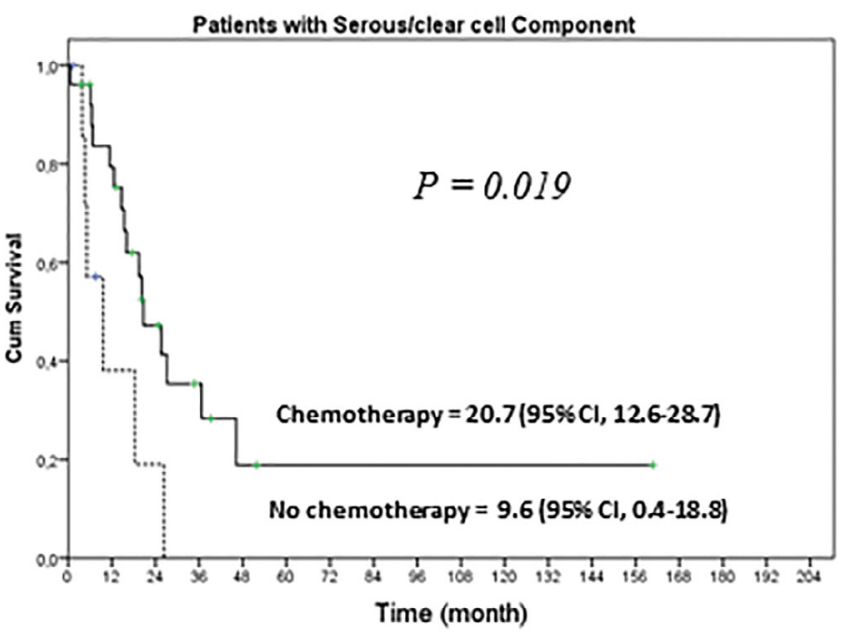

Heterologous subtype and unreported sarcomatous component occurred more frequently in UCS patients compared to OCS patients. The most common sarcomatous component in UCS patients was leiomyosarcoma, and chondrosarcoma was the most common sarcomatous component in OCS patients. The second most common sarcomatous component was rhabdomyosarcoma for both sites. The most common epithelial component in OCS and UCS patients were serous papillary carcinoma and endometrioid carcinoma, respectively. The distributions of the epithelial components of the carcinosarcomas were similar to the primary pure epithelial ovarian and uterine tumors. These findings about the frequency of epithelial and sarcomatous components were similar to the results presented in the current literature $(1,5)$.

b

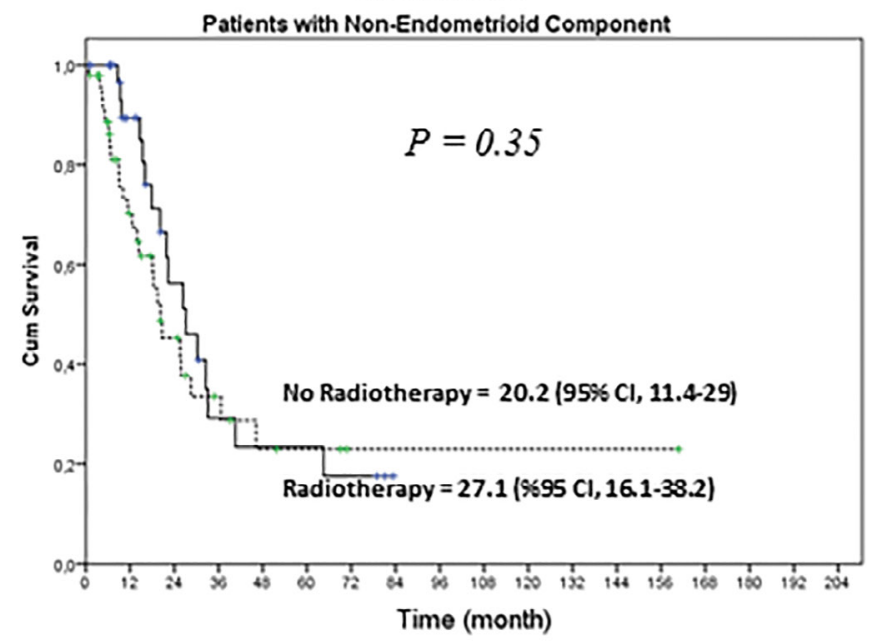

d Overall Survival

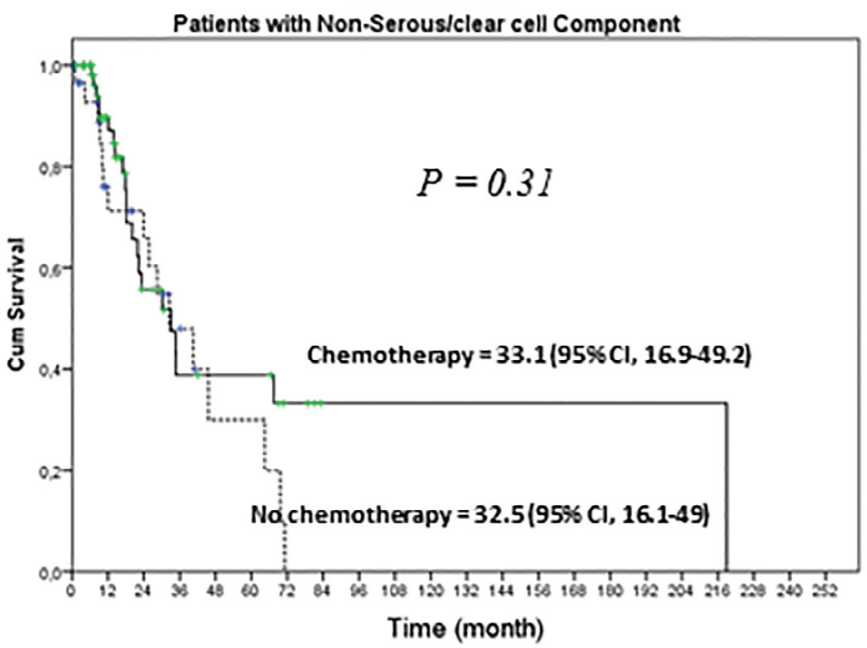

FIG. 3. a-d. Adjuvant radiotherapy significantly prolonged the median overall survival in patients with endometrioid component (a). Adjuvant radiotherapy had no beneficial effect on the survival of patients with non-endometrioid component (b) Patients with serous/clear cell component who received adjuvant chemotherapy had significantly longer median overall survival (c). In patients with non-serous/clear cell component, adjuvant chemotherapy had no beneficial effect on survival $(d)$. 
Age was not a prognostic factor for all patients. However, it was a prognostic factor for UCS patients. In Thasom et al. (7) also reported that age is a prognostic factor for UCS patients. In addition, Rauh-Hain et al. (8) found age to be a prognostic factor for OCS patients.

In the current study, the stage was one of the most important prognostic factors. The prognostic effect of the stage was more prominent in OCS patients. At an early stage, OCS patients had a more favorable prognosis compared to stage I-II UCS patients; however, at stage III, UCS patients tended to have worse prognosis. Similar to our findings, Garg et al. (9) found that patients with localized OCS have longer survival, although this finding was not statistically significant, compared to patients with localized UCS (129 months vs. 82 months, $p=0.11$ ); however, patients with OCS have significantly poor survival outcomes for regional disease ( 18 months vs. 28 months, $p=0.03$ ) and distant disease (10 months vs. 14 months, $\mathrm{p}=0.001)$.

Although endometrioid component was not a prognostic factor, it appeared to be a predictive factor for the efficacy of RT. Moreover, in both the univariate and multivariate analyses of UCS patients, CT did not appear to be an efficient treatment. Uterine carcinosarcoma patients with serous/clear cell component have poor prognosis. Athavale et al. (10) reported serous element as a poor prognostic factor for OCS patients. George et al. (11) reported that among the histological features of UCS, the epithelial tumor could influence the clinical course. In the current study, patients with serous/clear cell component who received CT had significantly better survival. These findings suggest that, similar to the most commonly seen pure epithelial uterine tumors, patients with UCS may benefit from RT or CT differently according to their epithelial components.

Although heterologous carcinosarcomas were reported as a negative prognostic factor in many studies, sarcomatous subtypes were not found to be a prognostic factor in others $(2,3,12-15)$. In this study, we also did not find sarcomatous subtypes to be a prognostic factor. The effect of RT was similar for the different sarcomatous subtypes. However, CT was an efficient adjuvant therapy in heterologous carcinosarcomas in our study. In many studies, a relationship between sarcomatous components and chemotherapy efficacy has not been shown $(2,3,16)$. However, patient numbers in the previous studies were lower than our sample size.

In our study, the efficacy of adjuvant therapy has been demonstrated clearly. In multivariate analysis performed for all patients, while CT was found to be an effective treatment option, RT was not. For UCS patients, while RT had a significant effect, CT did not. Our findings, rather than being inconsistent, suggest that the efficiency of adjuvant treatment options may be related to the primary tumor location, and the epithelial and sarcomatous elements. In our analysis, RCT and CT had similar OS outcomes. Because RCT did not provide better median OS in UCS patients with serous/clear cell component, in those patients, CT could be efficient at all stages whereas RT may have a limited indication for serous uterine carcinoma. In a Phase III randomized EORTC study, it suggested that adjuvant radiotherapy reduces local recurrence but do not improve survival outcome in stage I-II UCS (17). Cantrell et al. (18) retrospectively analyzed 111 stage I-II UCS patients (similar to our results), and they report that in a multivariate analysis, adjuvant CT had better PFS $(p=0.003)$ and OS $(p=0.058)$ than RT. Their findings were similar to ours. Tanner et al. found that patients with stage III-IV UCS who underwent cytoreductive surgery and an application of CT plus RT did not have a survival advantage over CT alone. However, these studies did not stratify their analyses according to histological subtypes.

In this study, patients who underwent pelvic \pm paraaortic lymph node dissection and patients who did not had similar OS. Patients with macroscopic residual disease after surgery had significantly worse OS. Tanner et al. (19) showed that complete resection followed by adjuvant treatment is the most important predictor for OS in patients with stage III-IV UCS. Doo et al. (20) reported that the degree of surgical cytoreduction is related to survival outcome and longest PFS and OS are achieved by non-visible disease after upfront surgical treatment in OCS. Similarly, we showed that complete resection was associated with improved survival on OCS patients in our previous study (15). Moreover, the absence of a relationship between the surgical procedure and OS is noteworthy. Together with this finding, the survival outcomes of patients receiving $\mathrm{RCT}$ are no better than patients receiving $\mathrm{RT}$ or chemotherapy alone; indicating that aggressive treatment approaches may not lead to better outcomes. In gynecological $\mathrm{CS}$, to avoid post-surgical macroscopic residual disease, neoadjuvant CT may be a preferential treatment option.

Our study has some limitations. First, the data were gathered from different centers using different RT and CT protocols. Due to the low number of patients, the different RT and CT protocols could not be analyzed. The lack of standardized surgery is another important weakness of this study. Adjuvant therapy options were not standardized among centers and were individual. In this multi-institutional study, centralized pathology review would be important and would improve the impact of our finding.

In conclusion, gynecological carcinosarcomas have different clinical and prognostic features according to their primary location. The serous/clear cell component of carcinosarcomas is a prognostic factor. Serous/clear cell and endometrioid components may be predictive factors for the efficacy of adjuvant CT or RT in patients with uterine and ovarian carcinosarcomas. Heterologous carcinosarcomas may benefit more from adjuvant CT. To the best of our knowledge, this current study is the first to report that epithelial and sarcomatous components are predictive factors for RT and CT. 
Ethics Committee Approval: Ethics committee approval was received for this study from the ethics comiittee of Trakya University School of Medicine.

\section{Informed Consent: N/A.}

Peer-review: Externally peer-reviewed.

Author contributions: Concept - İ.Ç. B.E.; Design - İ.Ç., T.Ö, B.E., E.T.; Supervision - İ.Ç., T.Ö.; Resource - M.Ö, D.G, A.G., Ö.B.; Materials - I.Ç., Y.Y., M.Ş., N.Ö.; Data Collection and/or Processing - İ.Ç, T.Ö, E.T., T.Ö., B.Y., M.Ö., D.G., M.Ş., N.Ö., B.Y., Ö.T., Y.G., S.M., B.Ö., A.A., B.E., M.B.H., E.A., A.S.; Analysis and/ or Interpretation - İ.Ç., B.E., T.Ö.; Literature Search - E.T., M.B.H., S.M., M.Ö.; Writing - İ.Ç., B.E., M.B.H., E.T., D.G., Y.G., S.M.; Critical Reviews - İ.Ç., E.A., A.S., B.E.

Acknowledgements: Authors would like to thank Dr. Osman Köstek for help of editing the manuscript.

Conflict of Interest: No conflict of interest was declared by the authors.

Financial Disclosure: The authors declared that this study has received no financial support.

\section{REFERENCES}

1. Soslow RA. Mixed Müllerian tumors of the female genital tract. Surg Pathol Clin 2009;2:707-30. [CrossRef]

2. Pacaut C, Bourmaud A, Rivoirard R, Moriceau G, Guy JB, Collard O, et al. Uterine and ovary carcinosarcomas: outcome, prognosis factors, and adjuvant therapy. Am J Clin Oncol 2015;38:272-7. [CrossRef]

3. Ariyoshi K, Kawauchi S, Kaku T, Nakano H, Tsuneyoshi M. Prognostic factors in ovarian carcinosarcoma: a clinicopathological and immunohistochemical analysis of 23 cases. Histopathology 2000;37:427-36 [CrossRef]

4. del Carmen MG, Birrer M, Schorge JO. Carcinosarcoma of the ovary: a review of the literature. Gynecol Oncol 2012;125:271-7. [CrossRef]

5. Kernochan LE, Garcia RL. Carcinosarcomas (malignant mixed Mullerian tumor) of the uterus: advances in elucidation of biologic and clinical characteristics. J Natl Compr Canc Netw 2009; 7:550-6; quiz 7.

6. Erickson BK, Doo DW, Zhang B, Huh WK, Leath CA 3rd. Black race independently predicts worse survival in uterine carcinosarcoma. Gynecol Oncol 2014;133:238-41. [CrossRef]

7. Inthasorn P, Carter J, Valmadre S, Beale P, Russell P, Dalrymple C. Analysis of clinicopathologic factors in malignant mixed Mullerian tumors of the uterine corpus. Int J Gynecol Cancer 2002;12:348-53. [CrossRef]

8. Rauh-Hain JA, Diver EJ, Clemmer JT, Bradford LS, Clark RM, Growdon WB, et al. Carcinosarcoma of the ovary compared to papillary serous ovarian carcinoma: a SEER analysis. Gynecol Oncol 131:46-51. [CrossRef]

9. Garg G, Shah JP, Kumar S, Bryant CS, Munkarah A, Morris RT. Ovarian and uterine carcinosarcomas: a comparative analysis of prognostic variables and survival outcomes. Int J Gynecol Cancer 2010;20:888-94. [CrossRef]

10. Athavale R, Thomakos N, Godfrey K, Kew F, Cross P, de Barros Lopes A, et al. The effect of epithelial and stromal tumor components on FIGO stage III and IV ovarian carcinosarcomas treated with primary surgery and chemotherapy. Int J Gynecol Cancer 2007; 17:1025-30. [CrossRef]

11. George E, Lillemoe TJ, Twiggs LB, Perrone T. Malignant mixed mullerian tumor versus high-grade endometrial carcinoma and aggressive variants of endometrial carcinoma: a comparative analysis of survival. Int J Gynecol Pathol 1995;14:39-44. [CrossRef]

12. Sood AK, Sorosky JI, Gelder MS, Buller RE, Anderson B, Wilkinson EJ, et al. Primary ovarian sarcoma: analysis of prognostic variables and the role of surgical cytoreduction. Cancer 1998;82:1731-7. [CrossRef]

13. Chang J, Sharpe JC, A'Hern RP, Fisher C, Blake P, Shepherd J, et al. Carcinosarcoma of the ovary: incidence, prognosis, treatment and survival of patients. Ann Oncol 1995;6:755-8.

14. Morrow CP, d'Ablaing G, Brady LW, Blessing JA, Hreshchyshyn MM. A clinical and pathologic study of 30 cases of malignant mixed mullerian epithelial and mesenchymal ovarian tumors: a Gynecologic Oncology Group study. Gynecol Oncol 1984;18:278-92. [CrossRef]

15. Cicin I, Saip P, Eralp Y, Selam M, Topuz S, Ozluk Y, et al. Ovarian carcinosarcomas: clinicopathological prognostic factors and evaluation of chemotherapy regimens containing platinum. Gynecol Oncol 2008;108:136-40. [CrossRef]

16. Kunkel J, Peng Y, Tao Y, Krigman H, Cao D. Presence of a sarcomatous component outside the ovary is an adverse prognostic factor for primary ovarian malignant mixed mesodermal/mullerian tumors: a clinicopathologic study of 47 cases. Am J Surg Pathol 2012;36:831-7. [CrossRef]

17. Reed NS, Mangioni C, Malmström H, Scarfone G, Poveda A, Pecorelli S, et al. Phase III randomised study to evaluate the role of adjuvant pelvic radiotherapy in the treatment of uterine sarcomas stages I and II: an European Organisation for Research and Treatment of Cancer Gynaecological Cancer Group Study (protocol 55874). Eur J Cancer 2008;44:808-18. [CrossRef]

18. Cantrell LA, Havrilesky L, Moore DT, O’Malley D, Liotta M, Secord AA, et al. A multi-institutional cohort study of adjuvant therapy in stage I-II uterine carcinosarcoma. Gynecol Oncol 2012;127:22-6. [CrossRef]

19. Tanner EJ, Leitao MM Jr, Garg K, Chi DS, Sonoda Y, Gardner GJ, et al. The role of cytoreductive surgery for newly diagnosed advanced-stage uterine carcinosarcoma. Gynecol Oncol 2011;123:548-52. [CrossRef]

20. Doo DW, Erickson BK, Arend RC, Conner MG, Huh WK, Leath CA 3rd. Radical surgical cytoreduction in the treatment of ovarian carcinosarcoma. Gynecol Oncol 2014;133:234-7. [CrossRef] 